\title{
Radial Flux High Temperature Superconductor Motor using Bulk Trapped Field Magnets
}

\author{
X. Feng*, G. Gao*, K. Davey**, M. Werst**, R. Hebner**, R. Weinstein***, D. Parks***, and R. Sawh*** \\ * R\&D Center, Teco-Westinghouse Motor Company, Round Rock, TX 78681, USA \\ ** Center for Electromechanics, University of Texas, Austin, TX 78758, USA \\ ***Texas Center for Superconductivity, and Physics Dept., University of Houston, Houston, TX 77204, USA
}

\begin{abstract}
This team has designed, built, and successfully run a radial flux high temperature superconductor (HTS) trapped field magnet (TFM) motor. The motor was run in a zero field cooled mode. This paper conveys several lessons learned about the use of TFMs in motor and generator applications. The state of the art of bulk TFM material is reviewed. Attention is given to the difficulties of activating TFMs in a practical machine design. The difficulties are based upon (i) the cooling time needed to field-cool the TFMs; (ii) the maximum field which can be produced at the rotor using only MMF on the stator; and (iii) the maximum time for which the stator current can be high without sustaining insulation damage. This is followed by a prediction of improvements that can be expected using two significant departures from a conventional three-phase permanent magnet design. Both departures involve the addition of MMF on the rotor using a hybrid wound rotor design. Finally attention is given to predicting performance when no special activation is employed.
\end{abstract}

\section{INTRODUCTION}

The first question demanding attention is "Why bother with bulk HTS trapped field magnet material?" The favored bulk HTS material is $\mathrm{YBa}_{2} \mathrm{Cu}_{3} \mathrm{O}_{\mathrm{y}}$, and it has a critical current of up to $325 \mathrm{kA} / \mathrm{cm}^{2}$, at $77^{\circ} \mathrm{K}$ and fields of 1 Tesla [1]. A practical TFM which can be mass-produced achieves a current density of $300 \mathrm{kA} / \mathrm{cm}^{2}$ [2], which results in a trapped field of 2.3 Tesla in air using a TFM only $2 \mathrm{~cm}$ in diameter. This TFM requires no cladding.

Thin film HTS tapes on the other hand require considerable cladding. Fig. 1 shows a typical thin film coated conductor assembly [3]. The support layers are 129 $\mu \mathrm{m}$ thick. Present currents in thin film YBCO are $\mathrm{J}_{\mathrm{c}} \sim 1.2$ $\mathrm{MA} / \mathrm{cm}^{2}$ at $77 \mathrm{~K}$ and zero applied field, but this decreases to $\mathrm{J}_{\mathrm{c}} \sim 200 \mathrm{kA} / \mathrm{cm}^{2}$ at 1 Tesla. The $129 \mu \mathrm{m}$ thickness of the support reduces the effective $\mathrm{J}_{\mathrm{c}}$ to only $\sim 3.1 \mathrm{kA} / \mathrm{cm}^{2}$. Thus at the field of interest, $\sim 1$ Tesla, the effective (engineering) value of $\mathrm{J}_{\mathrm{c}}$ in a TFM is about 100 times that of coated conductors.

Critical current density increases by about a factor of two for every $12^{\circ} \mathrm{K}$ dropped below $77 \mathrm{~K}$. One way of quantifying this is to measure the maximum achievable field at the surface of a $2 \mathrm{~cm}$ diameter TFM puck, $0.8 \mathrm{~cm}$ thick, in air (TABLE I).

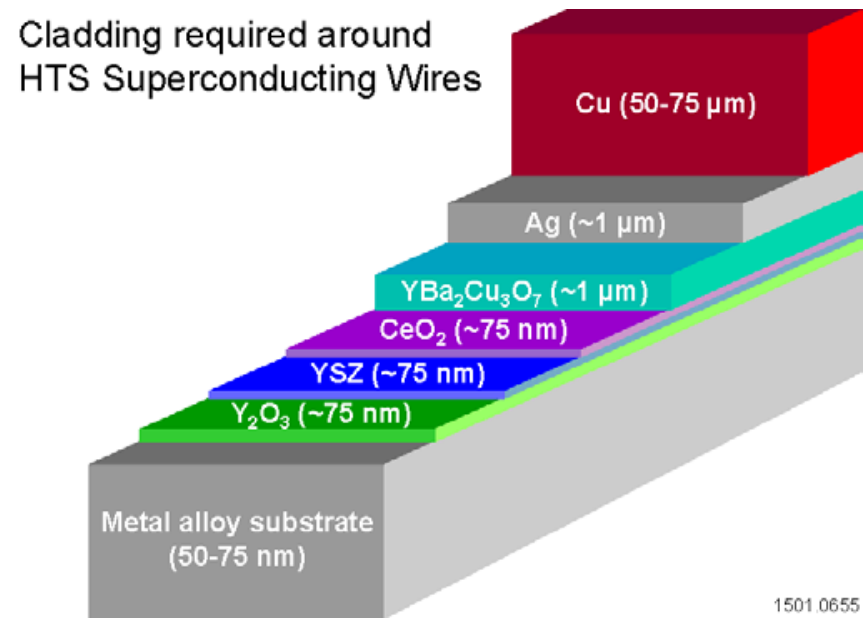

Figure 1. The cladding required around 2nd generation HTS YBCO wire leads to an equivalent current density of $201 \mathrm{MA} / \mathrm{m}^{2}$.

TABLE I

FIELD INCREASE WITH TEMPERATURE

\begin{tabular}{|c|c|}
\hline Temperature $\left({ }^{\circ} \mathbf{K}\right)$ & Field in air (T) \\
\hline 77 & 2.3 \\
\hline 65 & 5.1 \\
\hline 50 & 10.4 \\
\hline
\end{tabular}

\section{DiSADVANTAGES OF TFMS}

Although bulk YBCO has a conservative 100-fold advantage over HTS wire, it has three disadvantages. The first is that the material must be grown as a quasi-crystal, called a "grain." The second is that the material must be activated, an event that is analogous to magnetizing a permanent magnet. The third is that the field decreases with time.

Regarding the first disadvantage, the method of melttexturing [4] routinely produces excellent single grains $\sim 2$ $\mathrm{cm}$ in diameter at values of $\mathrm{J}_{\mathrm{c}}$ quoted above, and state-ofthe-art is approaching $3 \mathrm{~cm}$. Because maximum TFM field 
is $\mathrm{B}_{\max } \propto \mathrm{J}_{\mathrm{c}} \mathrm{x}$ diameter, and $2 \mathrm{~cm}$ grain diameter provides $\mathrm{B}_{\max } \sim 2.3$ Tesla in air, the $3 \mathrm{~cm}$ diameter grains are expected to provide $\mathrm{B}_{\max } \sim 2.7$ Tesla.

TFMs can be cast or cut to desired shapes, and can be machined to 0.005 inch accuracy. Hexagons are a popular shape since they lend themselves to large tiled arrangements. The difficulty is the interface between two tiles. Because it is not superconducting, the current within the TFM must lie within the perimeter of the puck. Some attention has been given to joining layers between TFMs, but current flow across the interface remains limited. This restriction makes a bulk YBCO array analogous to a number of strong, but nevertheless small magnetic dipoles, and limits the use of arrays in practical applications.

The second disadvantage is the activation requirement. Bulk TFMs are capable of trapping fields in excess of $17 \mathrm{~T}$ [5], but this state is not easy to achieve. Suppose a YBCO puck is exposed to a magnetic field (applied parallel to the $\mathrm{c}$-axis) equal to or greater than the largest $\mathrm{B}$ field that can be supported by a current density of $\mathrm{J}_{\mathrm{c}}$ throughout the puck. For $2 \mathrm{~cm}$ YBCO TFMs prepared using U/n irradiation, this value is $2.3 \mathrm{~T}$. If the material is exposed to this field, cooled, and then the source field removed, a current density of $J_{c}$ will reside throughout the puck, which will have a peak field of $2.3 \mathrm{~T}$, a state of full activation. A current density of the same value resides in the puck when it is exposed to a $1 \mathrm{~T}$ field during activation, but only a portion of the puck will have current, and the remainder will have none. If the material is already cooled before field is applied, the TFM can only be fully activated by exposing it to a field of twice the desired final value. If the material can be cooled after exposure to the desired magnetic field density, the full value of the activating field can be used to generate torque.

The trapped field decreases with time at a rate of typically $4 \%$ per decade of time. For example, a field which is $3 \mathrm{~T} 10$ minutes after activation will be $\sim 2.88 \mathrm{~T} 100$ minutes after activation. This means that the field does not drop below $80 \%$ of its initial value in practical equipment life-times. However, if such a field decrease is not palatable, a method of essentially stopping the decrease of trapped field has been developed [6]. This is accomplished by decreasing the temperature of the TFM by $\sim 4 \mathrm{~K}$, after activation.

In the discussion which follows, assume a permanent magnet type motor with a conventional three phase winding on the stator and TFM material in place of the permanent magnet rotor. One motivation for this configuration is that the TFM can be cooled easily through the surrounding steel and contained with the same. Field cooling (cooling after exposure to the desired field level) is favored.

\section{ACTIVATION FROM STATOR}

Obviously, activating the TFM in an HTS motor is most easily accomplished using windings already in place (e.g. stator in Fig. 2). The task amounts to balancing adiabatic heating on the stator with cooling time on the rotor. This team devised a cooling scheme for the motor construction,

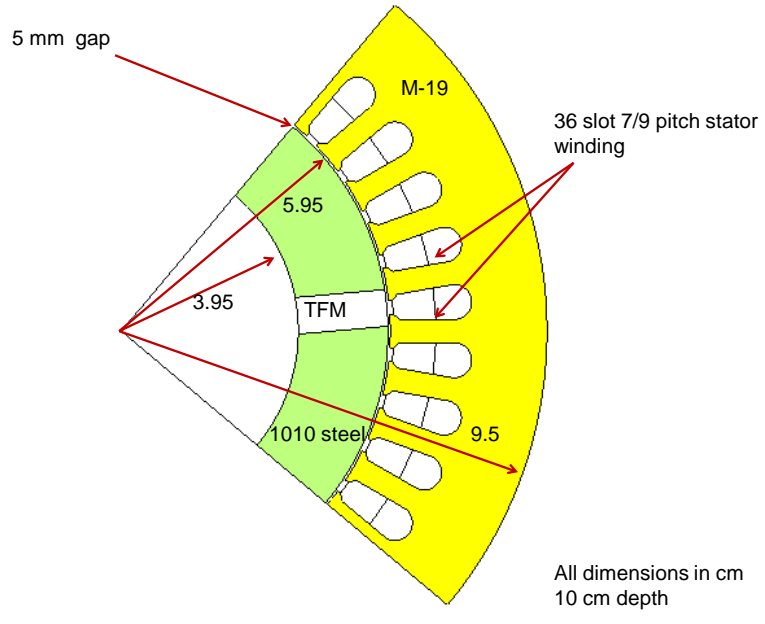

Figure 2. Baseline geometry for a four pole radial flux motor.

to send liquid $\mathrm{N}_{2}$ through the rotor shaft and to the interface between the TFM and rotor steel. A transient boundary element analysis was performed to predict the time required to cool this structure using liquid $\mathrm{N}_{2}$ (Fig. 3). The thermal analysis indicates that about $10-20 \mathrm{~s}$ are required to cool the TFM from $93 \mathrm{~K}$ to $77+\mathrm{K}$ and thus trap the field exposure.

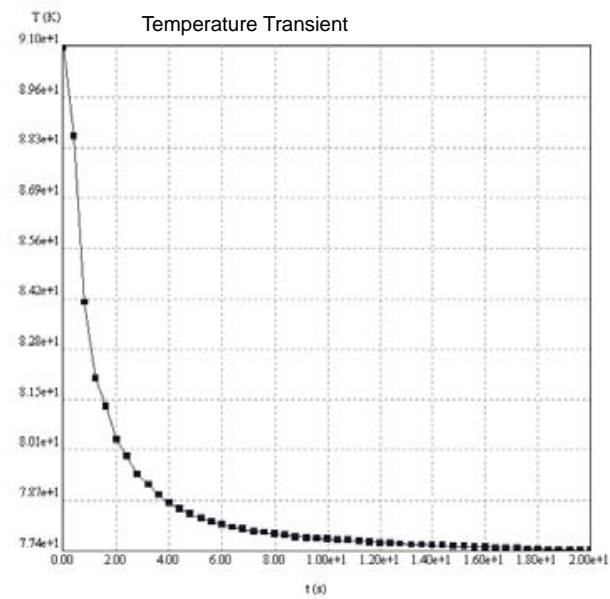

Figure 3. Transient temperature profile with time on the TFM puck when cooled with liquid N2.

What current can be sent through the stator without compromising the insulation? To simplify the analysis, consider what happens to the temperature on the stator winding if it is used to activate the TFM. If the stator has a cross-section area A with depth $\mathrm{L}$ with current density $\mathrm{J}$ and local conductivity $\sigma$, the ohmic power dissipation is

$$
P=(J \cdot A)^{2} \frac{L}{\sigma A}
$$

Adiabatic heating of the stator relates the power dissipation in time $\delta$ t to the stator (copper) density $\rho$, the specific heat constant $\mathrm{C}_{\mathrm{p}}$, and the temperature rise $\Delta \mathrm{T}$ as 


$$
P \delta t=\rho C_{p}(A L) \Delta T
$$

Combining (1) and (2) shows a relationship on the maximum allowed current density which is independent of the dimensions $\mathrm{L}$ and $\mathrm{A}$,

$$
J=\sqrt{\frac{\rho \sigma C_{p} \cdot \Delta T}{\delta t}}
$$

Type $\mathrm{H}$ insulation limits the temperature on the stator to $180^{\circ} \mathrm{C}$. Assume a working industrial environment with ambient temperature of $50^{\circ} \mathrm{C}$. Equation (3) allows a current density of $28 \mathrm{MA} / \mathrm{m}^{2}$. If a layered puck array is employed as in the lower inset of Fig. 4 (0.8 cm thick), a B field on the TFM array of only $0.67 \mathrm{~T}$ can be achieved (Fig. 5).



Figure 4. Field expected in the rotor TFM assuming an allowed $130{ }^{\circ} \mathrm{C}$ rise.



130 degree temperature rise for $20 \mathrm{~s}$ with a $78 \%$ packing factor gives $\mathrm{J}=2.8 \mathrm{e} 07 \mathrm{~A} / \mathrm{m}^{2}$

We require $18 \mathrm{e} 07 \mathrm{~A} / \mathrm{m}^{2}$ to get to $1.6 \mathrm{~T}$

Figure 5. B field across the TFM puck array using the conditions and array shown in Figure 4.
The primary problem is saturation in the stator, not limitations on thermal rise. Precooling the stator allows a substantial improvement in the current density that can be employed on the stator (Fig. 6).

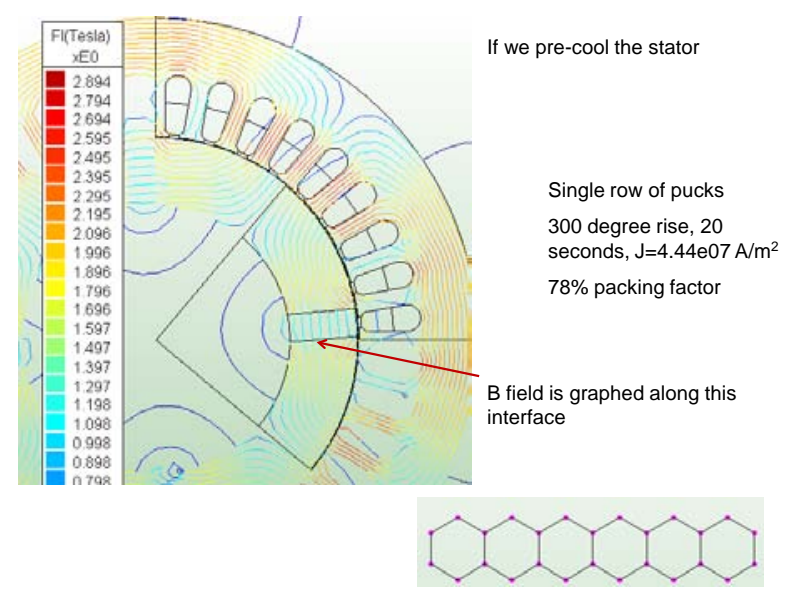

Figure 6. Magnetic field expected assuming a single layer TFM array and a 300 degree thermal rise in the stator.

With the higher stator current, the steel becomes quite saturated. Under these stressed conditions, the field achievable (Fig. 7) is not superior to that from a conventional $\mathrm{NdFeB}$ rotor except near the edges of the array where the field accentuates due to stator and rotor saturation.

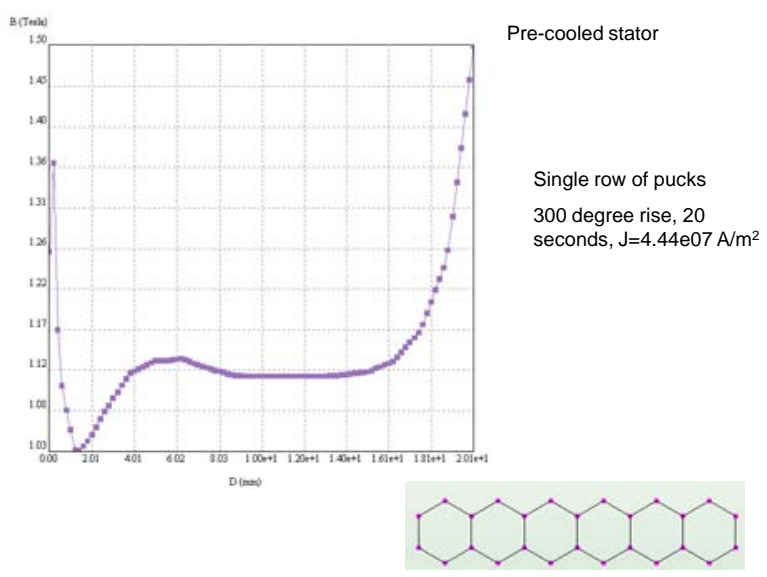

Figure 7. B field across the TFM array using the conditions cited in Figure 6.

Before exploring rotor excitation options, it should be noted that research with larger diameter TFM pucks may have a significant impact on activation requirements. A 1 $\mathrm{cm}$ hole can be drilled through a $3 \mathrm{~cm}$ diameter puck; the activation field of the modified $3 \mathrm{~cm}$ puck can be essentially the same as that of a $2 \mathrm{~cm}$ puck, but the larger puck allows cooling through the center orifice, and provides a more uniform field, with increased flux. This should drop the cooling time and increase the average field. 


\section{ACTIVATION FROM THE ROTOR}

Transferring the MMF for activating the TFM to the rotor suffers the substantial disadvantage of requiring a slip ring coupling and has a commensurate cost for coils that are only used during a fraction of the useful life of the motor.

Figure 8 shows a configuration suitable for generating a field in excess of 2T over the full TFM array, as shown in Fig. 9. The thickness of the region annotated YBCO has been increased to accommodate a dewar extending into the air gap. Having MMF on the rotor may be necessary to activate at high field levels. Two alternative embodiments of this design are being investigated. The first involves a tape wound steel core in place of the copper, and the second involves a self excited generator to achieve the high fields desirable for activation.

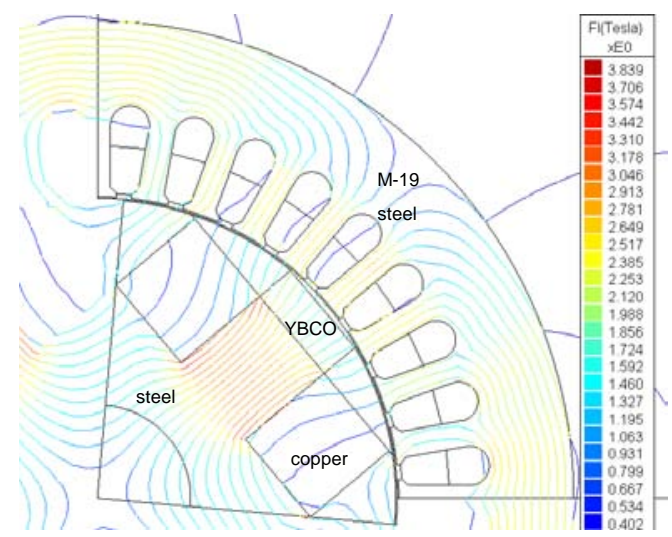

Figure 8. Possible configuration for TFM activation from the rotor.

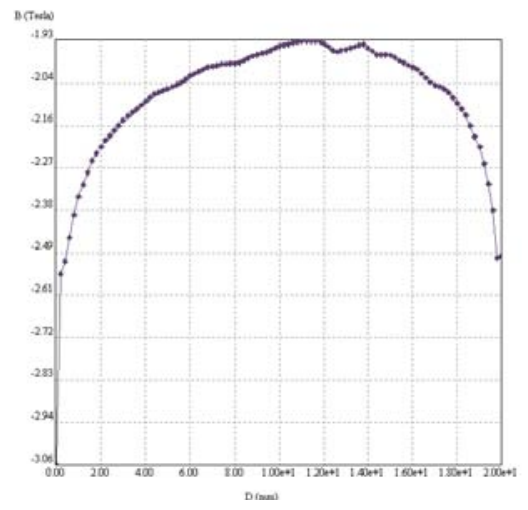

Figure 9. Magnetic field density along the interface of the TFM in Figure 8.

\section{RunNing Without Activation}

HTS TFM motors will run successfully without a special activation pulse, albeit with a small torque. This is sometimes referred to as the zero field cooled condition. Analysis of the device in this mode is best approached using the Bean model [7]. One technique tested by this team is to replace the TFM pucks with voltage fed coils [8].
The problem is complicated by the saliency of the rotor. The non-zero reluctance torque has a period of $22.5^{\circ}$. Fig. 10 shows the predicted torque as a function of rotor angle just due to the induced currents in the TFM. The $10^{\circ}$ repetition rate is due to the spacing of the stator slots. The particular geometry encourages localized slot leakage and the radial strips of TFM material responds to this effect. The current in the TFM will only rise to the level sufficient to repel the flux from the bulk YBCO. Only a small fraction of the flux will go through the gap occupied by the TFM, which magnetically looks like air. The current in the TFM pucks is about 8 times that in one-half of one stator slot, but it is ineffective in coupling to the stator. A torque less than $0.5 \mathrm{~N}-\mathrm{m}$ is the result.

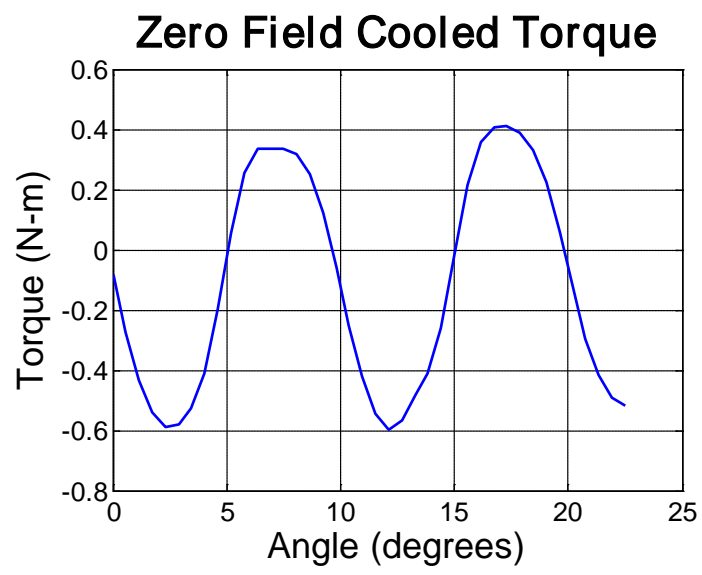

Figure 10. Torque created by the TFM when the puck is zero field cooled.

\section{High TEMPERATURE SUPERCONDUCTOR MOTOR USING BULK TRAPPED FIELD MAGNETS}

Because they have now been demonstrated to produce very large fields and they can be pulse activated, they offer the promise of high power density for electrical machine application and therefore, low cost and high performance can be obtained with high temperature superconductor machine using bulk trapped field magnet. One investigation shows a comparison of total volume, active rotor material, cost of active material and machine efficiency of Permanent Magnet (PM) machine, High Temperature Superconductor (HTSC) machine and High Temperature Superconductor Trapped Field Magnet (TFM) Bulk machine (TABLE II).

TABLE II

COMPARISON OF THE PERFORMANCE AND MATERIAL COSTS FOR $1 \mathrm{MW}$ MOTOR BETWEEN PM, TFMS AND HTSC MACHINES

\begin{tabular}{|c|c|c|c|}
\hline Parameter & PM Machine & TFM Bulk & HTSC Wire \\
\hline $\begin{array}{c}\text { Total Volume } \\
\mathbf{( m m}^{\mathbf{3}} \mathbf{)}\end{array}$ & $114 * 10^{6}$ & $33 * 10^{6}$ & $17.9 * 10^{6}$ \\
\hline $\begin{array}{c}\text { Active Rotor } \\
\text { Material (mm }\end{array}$ & $\left.7.26 * 10^{6}\right)$ & $1.92 * 10^{6}$ & $0.54 * 10^{6}$ \\
\hline $\begin{array}{c}\text { Cost of } \\
\text { Material }\end{array}$ & $\$ 13.66 \mathrm{~K}$ & $\$ 5.265 \mathrm{~K}$ & $50.90 \mathrm{~K}$ \\
\hline Efficiency & $96.5 \%$ & $98.7 \%$ & $98.1 \%$ \\
\hline
\end{tabular}




\section{A. Prototype of High Temperature Superconductor Motor using Bulk Trapped Field Magnet}

As mentioned advantages of trapped field magnet bulk, the prototype of radial flux high temperature superconductor motor using bulk trapped field magnet has been developed in the same way that permanent magnets are used into permanent magnet electrical machines today. Major differences include the fact that the trapped magnet bulks in the motor must be activated or charged in situ, and it must be kept at cryogenic temperatures. After carefully evaluating various rotor configurations and performance analyses of permanent magnet electrical machine, an inner TFM rotor structure was used in the prototype design rather than a less-efficient a surface-mounted TFM rotor configuration. The configuration of radial flux high temperature superconductor synchronous machine using bulk trapped field magnet (TFM) is shown in Fig. 11. This approach was viewed as a step toward commercialization in comparison with earlier work [9].

\section{D Model of Demo Motor}

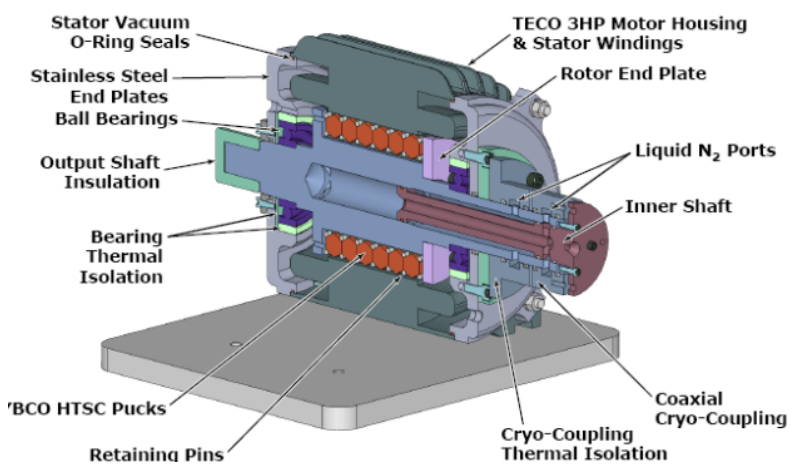

(a) 3D model of HTSC motor with TFM

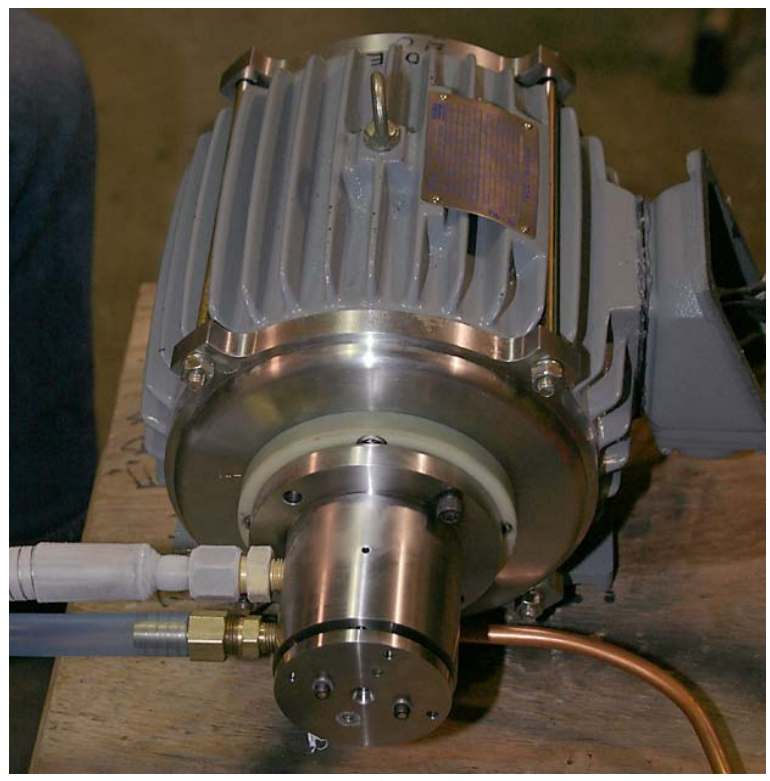

(b) Prototype of HTSC motor with TFM

Figure 11. High temperature superconductor motor using bulk trapped field magnet.

\section{B. Activation Tests}

Two zero field activation tests were performed: $50 \mathrm{~A}$ and 110 A dc excitation levels. Upon completion of the activation, the motor was immediately operated as a generator. The open circuit voltage measurements for both of these tests are shown in TABLE III.

TABLE III

MAGNET ACTIVATION TEST RESULTS

\begin{tabular}{|c|c|c|c|c|}
\hline $\begin{array}{c}\text { Activation } \\
\text { Type }\end{array}$ & $\begin{array}{c}\text { DC Current } \\
\text { (A) }\end{array}$ & Time (s) & RPM & $\begin{array}{c}\text { Output } \\
\text { Volts, rms } \\
\text { (V) }\end{array}$ \\
\hline Zero & 110 & 2 & 675 & 9.0 \\
\hline Zero & 50 & 10 & 721 & 20.5 \\
\hline Field & 28 & 60 & 740 & 14.6 \\
\hline Field & 8 & 600 & 730 & 15.7 \\
\hline
\end{tabular}

Two field activation tests were performed. Since the initial motor cool-down rate demonstrated good repeatability (Fig. 12), a 28 A, 60 s dc charge current was applied when the temperature in the rotor reached $-170^{\circ} \mathrm{C}$.



Figure 12. Motor cool down data showing timing planning for field activation tests

An open circuit voltage of $14.6 \mathrm{~V}$ rms was measured at the output of the motor. Motor output without activation, cold (residual magnetism only) was measured to be $1.6 \mathrm{~V}$ rms. Full activation of the pucks should have produced significantly more voltage. The discrepancy may be explained by the following possibilities:

1) The pucks are very likely at the upper temperature of field retention

2) The charging window may have been mistimed

3) The predictions assume a $100 \mathrm{~mm}$ length of continuous pucks and, due to puck shape, this overestimates the volume of puck material

4) Temperature differences within a puck array and among the four arrays

The only feedback on rotor temperature is a sheathed thermocouple probe in the iron in the center of a pole. The lowest temperature measurements from the rotor probe range from $-180^{\circ} \mathrm{C}$ to $-183^{\circ} \mathrm{C}$. The probe submersed in liquid nitrogen will read $-191^{\circ} \mathrm{C}$ suggesting an absolute temperature measurement error of $5^{\circ} \mathrm{C}$. To eliminate possibility number 2 above, a second attempt at field activation was performed using a lower current (8 A) 
TABLE IV

RMS MOTOR CURRENT DATA

\begin{tabular}{|c|c|c|c|c|c|c|c|}
\hline \multirow{2}{*}{ Frequency } & \multirow{2}{*}{ RPM* } & \multicolumn{2}{|c|}{ Phase A (Amps-rms) } & \multicolumn{2}{|c|}{ Phase B (Amps-rms) } & \multicolumn{2}{|c|}{ Phase C (Amps-rms) } \\
\hline & & Un-activated & Activated** & Un-activated & Activated** & Un-activated & Activated** \\
\hline 15 & 450 & 4.63 & 3.80 & 13.70 & 12.92 & 15.80 & 3.47 \\
\hline 20 & 600 & 4.89 & 4.56 & 16.48 & 10.65 & 14.67 & 8.57 \\
\hline 30 & 900 & 5.25 & 5.26 & 18.03 & 10.82 & 16.02 & 14.06 \\
\hline 40 & 1200 & 5.15 & 5.23 & 17.63 & 10.74 & 16.12 & 13.94 \\
\hline 50 & 1500 & 4.88 & 5.01 & 17.17 & 9.32 & 17.10 & 11.52 \\
\hline 60 & 1800 & 3.97 & 4.01 & 11.86 & 7.04 & 12.50 & 8.65 \\
\hline
\end{tabular}

* Rotor speed measured with Shimpo DT301 digital stroboscope

**8A DC field activation

for a much longer duration (600 s). The output voltage for a much lower charge current was comparable so mistiming was a relatively small contributor to the lower than expected output voltage.

Fig. 13 shows the open circuit voltage as a function of time as the motor warms. The cooling was turned off at time zero and the voltage begins to decay after about $50 \mathrm{~s}$. The voltage then decays over a period of $35 \mathrm{~s}$ to $4.1 \mathrm{~V} \mathrm{rms}$, not the level of the residual voltage of $1.6 \mathrm{~V}$ rms. This data suggests there is a slow decay of a small residual magnetic field as the system warms. This behavior has not yet been explained.

The level region in the first approximately 50 seconds suggests the rotor was in thermal equilibrium with its environment. It does not prove, however, that all of the pucks were at the same temperature. While it is clear that some of the pucks were at a low enough temperature to be activated, there the measured output voltage suggests that not all were.

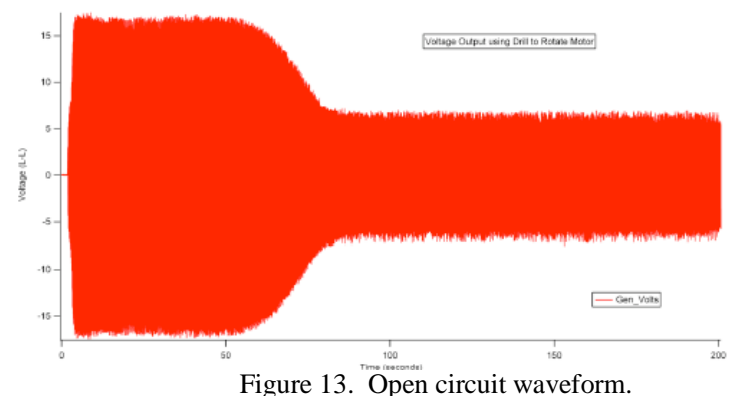

\section{Motor Operation Tests}

The motor was operated at $460 \mathrm{~V}$ in both activated and non-activated conditions from 15 to $60 \mathrm{~Hz}$. RMS phase currents are listed in Table IV for each operating frequency. Phase currents were measured using clamp around ammeters. The ac accuracy in $150 \mathrm{~A}$ range is $+/-1.5 \%$ of reading $+0.5 \mathrm{~A}$. Current waveforms were recorded using an oscilloscope. The phase A ammeter behaved erratically and therefore the data is suspect. The ammeter used during initial motor testing was less accurate by an unknown amount below $45 \mathrm{~Hz}$. Finally, there were likely small differences in temperatures within this data set. Both time at temperature and changing thermal losses due to changing rotor speed complicate the data interpretation with the limited temperature data available.

\section{Summary}

The motor was operated using $460 \mathrm{~V}$ with a $5 \mathrm{hp}$ motor drive at frequencies from 15 to $60 \mathrm{~Hz}$ in both the activated and non-activated states. The rms phase currents show a definite reduction across the frequency range when the pucks were activated. Synchronous operation was also verified at each frequency.

Operating the motor as a generator turned out to be the best diagnostic for determining puck activation. This data verifies activation of the pucks using both field activation and zero field activation. When operating as a generator, the voltages produced were significantly lower than predicted. This behavior can likely be explained and minimized with improved thermal management.

\section{CONCLUSIONS}

The effective use of TFM materials in electromechanical devices demands creative techniques for activating the bulk YBCO. Radial flux drives will require activation coils on the rotor to achieve field levels consistent with the potential of the material. Saturation limits the ability of the stator coils to achieve sufficiently high levels on the rotor. TFM devices can be replaced with zero resistance, zero voltage coils to determine how they will perform in zero field cooled activations.

\section{ACKNOWLEDGMENT}

The University of Houston group is supported in part by grants from the US Army Research office, and Welch Foundation, and the State of Texas via the Texas Center for Superconductivity at UH.

\section{REFERENCES}

[1] G. Fuchs, K. Nenkov, G. Krabbes, R. Weinstein, A. Gandini, R. Sawh, B. Mayes, and D. Parks, "Strongly enhanced irreversibility fields and Bose-glass behaviour in bulk YBCO with discontinuous columnar irradiation defects.” Supercond. Sci. Technol. 20 (2007) S197-S204.

[2] Roy Weinstein, Ravi Sawh, Yanru Ren, Michael Eisterer, Harald W. Weber, "The role of uranium chemistry and uranium fission in obtaining ultra-high Jc in textured Y123.” Supercond. Sci. Technol, 11 (1998) 959-962.

[3] Martin W. Rupich, Darren T. Verebelyl, Wei Zhang, Thomas Kodenkandath, and Xizoping Li, "Metalorganic Deposition of YBCO Films for Second-Generator High-Temperature Superconductor Wires,” Materials Research Journal, vol. 29, no. 8, 2004. 
[4] K. Salama, V. Selvamanickam, L. Gao and K. Sun, "High current density in bulk YBCO superconductor,” Appl. Phys. Lett., 54, 2352 (1989).

[5] M. Tomita and M. Murakami, "HTS bulk magnets that can trap over 17 Tesla at $29 \mathrm{~K}$,” Nature, 42, 517 (2003).

[6] R. Weinstein, J. Liu, Y. Ren, R-P Sawh, D. Parks, C. Foster, and V. Obot, Invited Paper, "Very High Trapped Fields: Cracking, Creep, and Pinning Centers," Proc. of 10th Anniversary HTS Workshop on Physics, Materials and Applications, Houston, edited by W.K. Chu, D. Gubser, and K.A. Müller, World Scientific Press, p. 625 (1996)
[7] C. Bean, Revs. Mod. Phys., 36, 31 (1964).

[8] Kent R. Davey, Roy Weinstein, and Ravi Sawh, "Development and analysis of YBCO tapped field magnets in elecromechanical devices," IEEE Transactions on Magnetics, Vol. 44, No. 6, June 2008, pp. 930-933.

[9] R. Weinstein, R. Sawh and A. Crapo, “An experimental Generator Using High Temperature Superconducting Quasi-Permanent Magnets”, IEEE Trans. Appl. Superconductivity, vol. 5, pp. 441 444, 1995. 